\title{
Percepción y práctica de la responsabilidad social empresarial: el caso de las pyme de Salinas, Santa Elena
}

\author{
Otto Suárez Rodríguez \\ Universidad Tecnológica Equinoccial, Sede Santa Elena (Ecuador) \\ otto.suarez@ute.edu.ec \\ Bruno Burgos Vera \\ Universidad Tecnológica Equinoccial, Sede Santa Elena (Ecuador) \\ bruno.burgos@ute.edu.ec
}

Fecha de presentación: 19 de octubre de 2015 • Fecha de aceptación: 11 de agosto de 2016

Artículo de investigación 


\section{Resumen}

Este trabajo busca determinar el grado de conocimiento y práctica de la responsabilidad social empresarial (RSE) en las pyme de Salinas, Santa Elena. Este centro turístico de la novel provincia de Santa Elena, en Ecuador, cuenta con un sector empresarial autóctono pequeño pero dinámico en aras de contribuir al desarrollo de su comunidad. Mediante encuestas aplicadas a las pyme, se valoraron cualitativamente indicadores de RSE. Los resultados indican que la RSE se practica más por los valores propios del empresario que por un esfuerzo consciente y sistemático, lo que limita su incidencia en el desarrollo de la comunidad.

Palabras clave: Responsabilidad social empresarial, pyme, desarrollo humano, Salinas, Ecuador.

JEL: M14: Cultura corporativa; responsabilidad social corporativa.

\section{Abstract}

This work seeks to determine the degree of knowledge and practice of corporate social responsibility (CSR) in SMEs in Salinas, Santa Elena. This tourist destination in the new province of Santa Elena in Ecuador has a small but dynamic local business sector in order to contribute to the development of its community. Through surveys applied to SMEs, CSR indicators were qualitatively assessed. The results indicate that CSR is more practiced by the entrepreneur's values than by a conscious and systematic effort, which limits its impact on the development of the community.

Keywords: Corporate Social Responsibility, SMEs, human development, Salinas.

JEL: M14: Corporate Culture; Diversity; Social Responsibility.

\section{Resumo}

Este trabalho procura determinar o grau de conhecimento e prática da Responsabilidade Social Empresarial (RSE) nas PMEs da Salinas, Santa Elena. Este centro turístico da novel província de Santa Elena em Equador, conta com um sector empresarial autóctono pequeno mas dinâmico em aras de contribuir ao desenvolvimento de sua comunidade. Mediante encuestas aplicadas às PMEs, valorizaram-se qualitativamente indicadores de RSE. Os resultados indicam que a RSE se pratica mais pelos valores próprios do empresário que por um esforço consciente e sistémico, o que limita sua incidência no desenvolvimento da comunidade.

Palavras-chave: Responsabilidade Social Empresarial, PMEs, desenvolvimento humano, Salinas.

JEL: M14: Cultura corporativa; Responsabilidade social. 


\section{Introducción}

1 contexto histórico-económico muestra fehacientemente que la proactividad organizativa y el crecimiento privado han sido factores de alto impacto en la sociedad. La empresa privada, en la producción de bienes, servicios, ideas, fuentes de trabajo, innovación, entre otras actividades, genera riqueza y contribuye de manera sustancial a la producción nacional y al desarrollo de todo conglomerado humano.

En este marco, la gestión de la responsabilidad social empresarial (RSE) se ha convertido en una estrategia que genera competitividad y beneficios para los accionistas, colaboradores, clientes, proveedores, la comunidad en general, así como para el medio en donde se desenvuelve la empresa; el término en inglés stakeholders es el usual para referirse a todas estas partes interesadas.

El Plan Nacional del Buen Vivir (SENPLADES 2013) reconoce en algunos ámbitos el rol de la RSE en el desarrollo nacional y la aportación que el sector productivo privado puede brindar como actor social clave. En esta línea, el presente estudio se centra en conocer cómo se aplica y percibe la RSE en el cantón Salinas, provincia de Santa Elena (Ecuador) y responde a una primera aproximación sobre los indicadores de RSE propuestos por Fraga, Herrera y Gómez (2015) para pyme del cantón Salinas.

Estos autores, a base de aportaciones anteriores, desarrollaron un conjunto de indicadores de RSE que, aplicados a las empresas, permite visualizar la existencia y la práctica de los principios de la RSE en la gestión empresarial y en la medida en que las empresas practican la RSE, los resultados se traducen en contribuciones al desarrollo humano de las comunidades donde actúan.

El estudio permite identificar los diferentes momentos en que la RSE se hace presente en la gestión empresarial: desde la planificación estratégica, 
implementación, hasta la medición y comunicación de resultados. La investigación se complementa con el sentir de la población respecto de cómo ven la gestión empresarial en cuanto al apoyo a la comunidad y protección del medioambiente que reciben de las pyme en su actividad diaria.

\section{Responsabilidad social empresarial y desarrollo humano}

Las teorías y conceptualizaciones relacionadas con la RSE son recientes; por ejemplo, Garriga y Melé (2004) sostienen que los intentos por generar un marco conceptual de la RSE inician hacia mediados del siglo XX; además sostienen la hipótesis de que las teorías y enfoques se relacionan con los beneficios, la política, las demandas sociales y los valores éticos.

Reflexionando sobre los aportes realizados en pro de la RSE, se cita a Mílton Friedman (1970), con su trabajo The Social Responsibility of Business is to Increase its Profits, quien sostiene que la empresa y la responsabilidad social son dos cosas separadas, es decir, la empresa es creada para generar riqueza y su rol es netamente económico y, más bien, debe ser el individuo (accionista) quien realice labor social, haciendo uso de las utilidades generadas por la empresa si a bien tuviere.

La reflexión anterior se la puede comparar con la tesis que mantenía Andrew Carnegie en su obra Wealth de 1889 (citado por Cancino y Morales 2008 , 9), quien mantenía que las personas que más tienen deberían compartir con aquellos que no hubieran alcanzado el bienestar debido. Este principio de filantropía (caridad) se transforma más tarde en las utilidades que comparte la empresa con sus empleados o colaboradores; acción que se enmarca en una gestión por proceso y no durante el desarrollo del proceso mismo; en consecuencia, se la ha considerado filantropía.

El enfoque contemporáneo de la RSE no está basado en la filantropía sino que se centra en el desarrollo de acciones durante el proceso de producción de la organización. En este sentido, Cancino y Morales (2008, 11) manifiestan que "Sin duda alguna, el que exista una visión más completa de lo que debe significar RSE hace que cada sociedad pueda evaluar de mejor forma el real aporte que desarrolla cada empresa sobre su entorno. Teniendo 
una visión más integral de RSE, y menos parcializada, los elementos a evaluar son más transparentes".

Howard Bowen (citado por Moon y Vogel 2008, 304), a menudo considerado el padre de la RSE, definió la responsabilidad social del empresario como las obligaciones que este tiene para aplicar aquellas políticas, tomar aquellas decisiones o seguir aquellas líneas de acción que son deseables en términos de los objetivos y valores de nuestra sociedad. De esta forma, la empresa y la sociedad quedan vinculadas de manera ineludible y sistemática, mientras que el gobierno establece el marco conductual del sector privado para servir a los fines sociales, por medio de la política pública de regulaciones e incentivos.

Según Canessa y García $(2005,31)$, “los beneficios de las prácticas socialmente responsables se pueden agrupar en tres grandes ventajas: el mejoramiento del desempeño financiero, la reducción de los costos operativos de las empresas y la mejora de la imagen de marca, y la reputación de la empresa". Por medio de estos fines netamente de interés empresarial, las empresas como actores sociales y su gran capacidad de impacto en el devenir de la sociedad, tienen también una gran responsabilidad frente a esta, pues el ámbito económico en el que actúan determina en gran medida las condiciones de vida y los niveles de bienestar de los miembros de la sociedad, conforme lo sostiene Barco Sousa (2015).

En este sentido, el vínculo entre RSE y desarrollo humano es insoslayable. En efecto, tal como lo concibe el Programa de las Naciones Unidas para el Desarrollo (PNUD 2015), el desarrollo humano busca el aumento de la riqueza de la vida humana en lugar de la riqueza de la economía. Se trata de un enfoque centrado en crear mejores oportunidades y posibilidades de elección para todas las personas. Por su parte, para Fraga, Herrera y Gómez (2015) el desarrollo humano es concebido como un proceso dirigido a ampliar las oportunidades de la gente para satisfacer sus necesidades fundamentales y, de esta forma, lograr mejores condiciones de vida; y encuentra en el quehacer empresarial uno de los medios para ampliar dichas oportunidades, pues es la empresa la que en gran medida satisface las demandas sociales y como tal está íntimamente ligada a la sociedad.

La norma ISO $26000(2010,11)$, concebida para ser una guía de la gestión de la RSE, establece que "es la responsabilidad de una organización ante 
los impactos de sus decisiones y actividades en la sociedad y el medioambiente, mediante un comportamiento ético y transparente que:

- Contribuya al desarrollo sostenible incluyendo la salud y el bienestar de la sociedad;

- Tome en consideración las expectativas de sus partes interesadas;

- Cumpla con la legislación aplicable y sea coherente con la normativa internacional de comportamiento; y

- Esté integrada en toda la organización y se lleve a la práctica en sus relaciones".

La normativa también plantea como parte de su gestión de RSE la rendición de cuentas no solo a sus colaboradores y accionistas; sino también a la sociedad y sus grupos de interés por impacto de las actividades y decisiones, asumir responsabilidades por el daño que se provoque a la comunidad y al medioambiente.

\section{Metodología}

Partiendo de los indicadores de RSE y de incidencia en el desarrollo humano identificados por Fraga, Herrera y Gómez (2015), se propone diagnosticar el aporte de las pyme del cantón Salinas a la RSE y discernir su impacto en el desarrollo del entorno y la comunidad en general, bajo la óptica del respeto por la naturaleza y el buen vivir.

Salinas es uno de los tres cantones que componen la provincia de Santa Elena en Ecuador. Económicamente depende principalmente de la actividad turística y la pesca artesanal. Considerando que Santa Elena es la provincia más nueva del Ecuador (creada en 2007), el sector empresarial propio de la provincia recién se está cimentando, toda vez que gran parte de la actividad productiva y de servicios ha dependido tradicionalmente de las grandes ciudades como Quito y Guayaquil. En este sentido, amerita conocer cómo el empresario de Salinas se adhiere a las prácticas socialmente responsables y en el proceso contribuye al desarrollo humano de su comunidad.

Se diseñaron dos encuestas, una para las pyme del cantón en cuanto a los indicadores de RSE e incidencia en el desarrollo humano, y otra para la población del cantón en cuanto a la percepción de las pyme y la RSE. 
De diferentes fuentes secundarias del Servicio de Rentas Internas (SRI), la Superintendencia de Compañías del cantón Salinas, la Cámara de Comercio Península de Santa Elena y el Ministerio de Industrias y Productividad, se pudo determinar un universo de 49 pyme que operan en el cantón Salinas. Considerando el pequeño número de empresas, se aplicó la encuesta a todo el universo. Sin embargo, luego de un primer trabajo de identificación en campo se estableció que solo 23 empresas estaban activas a la fecha de la encuesta.

El cuestionario aplicado a las empresas contiene 10 secciones de preguntas, de las cuales las cuatro primeras corresponden al tiempo de presencia de la empresa, el número de trabajadores, el número de trabajadores por sexo y el sector productivo al que pertenece. La quinta sección contiene 30 indicadores de $\operatorname{RSE}^{1}$ en donde las empresas tenían que responder si en su gestión existe o practica cada indicador (preguntas cerradas dicotómicas). Las cinco secciones finales buscan relacionar las aportaciones de las empresas a la RSE con el desarrollo humano.

En cuanto a los pobladores, a diferencia de las empresas, se realizó un muestreo donde las unidades a analizar fueron los hogares, para lo cual se los dividió en tres estratos: la cabecera cantonal Salinas (estrato I), la parroquia José Luis Tamayo (estrato II), y la parroquia Anconcito (estrato III). Esta estratificación se fundamentó en que los residentes de Salinas son de un estrato "medio+", con relativamente mejores condiciones de vida (disponibilidad de todos los servicios, calles y avenidas asfaltadas en su mayoría, planificación urbana, entre otros). Mientras que en José Luis Tamayo las condiciones de vida de sus residentes son medio o medio bajo (pocas calles y avenidas asfaltadas, poca planificación urbana, invasiones a terrenos baldíos, estructura informal de las viviendas, entre otros). Similares son las condiciones en Anconcito; sin embargo, al estar aislada del circuito Santa Elena-Libertad-Salinas, se diferencia su crecimiento y por ende su población. Sobre la base de lo antes detallado, se determinó la muestra según se detalla en la tabla 1.

1. Los indicadores del 1 al 7 corresponden al grupo de valores y transparencia; 8 al 10 a calidad de producción y servicios; 11 al 15 a actores internos; 16 al 20 a medioambiente; 21 al 26 a público externo; y 27 al 30 a gobierno y sociedad. 
Tabla 1

Número de viviendas y tamaño de la muestra de Salinas, José Luis Tamayo y Anconcito

\begin{tabular}{|l|c|c|c|}
\hline \multicolumn{1}{|c|}{ Estrato } & No. de viviendas* & No. de estrato & Tamaño de la muestra \\
\hline Salinas & 8.085 & $\mathrm{I}$ & 77 \\
\hline J. L. Tamayo & 5.376 & $\mathrm{II}$ & 52 \\
\hline Anconcito & 2.699 & $\mathrm{III}$ & 26 \\
\hline Total & 16.160 & & 155 \\
\hline
\end{tabular}

*Viviendas particulares ocupadas con personas presentes.

Fuente: VII Censo de Población y VI de Vivienda (INEC 2010).

Elaboración propia.

Para el cálculo del tamaño de la muestra estratificada se aplicó la fórmula de Cochran (1991) con un error estándar de 2\% y probabilidad de éxito de 0,5 , obteniéndose un tamaño de muestra de 155 viviendas.

$$
n=\frac{\left\{\sum N_{h} * p * q\right\}^{2}}{V^{2}+\sum\left\{N_{h} *(p * q)^{2}\right\}}
$$

Donde $n$ es el tamaño de la muestra, $h$ identifica al estrato, $N_{h}$ es la población de cada estrato, $p$ es la probabilidad máxima de éxito, $q$ es la probabilidad máxima de fracaso y $V^{2}$ es la varianza deseada.

Por medio del método de afijación proporcional se determinó el tamaño de muestra de cada estrato, aplicando la siguiente fórmula:

$$
n_{h}=n \frac{N_{h}}{N}
$$

Donde $n_{h}$ es el tamaño de la muestra del estrato h y $N$ es la población total del cantón Salinas (tabla 1).

Una vez calculado el tamaño de la muestra y repartida entre los diferentes estratos con afijación proporcional, se procedió a seleccionar las manzanas "unidades primarias de muestreo (UPM)" en cada estrato, usando números aleatorios. Posteriormente, se escogió al azar una vivienda al interior de cada manzana; vivienda que se convirtió en la unidad de información para la encuesta sobre la RSE. 
El cuestionario aplicado a la población incluyó entre las preguntas relevantes el conocimiento de la RSE, la percepción de los roles de la empresa y del sector público y la valoración que dan a la práctica empresarial tendiente a la responsabilidad social.

\section{Análisis de resultados y discusión}

En esta sección se presentan los principales resultados obtenidos de la aplicación de las encuesta a las pyme y población del cantón Salinas.

\section{Aporte de las pyme a la RSE}

La tabla 2 muestra el tiempo de presencia de las pyme en el cantón Salinas. Se puede apreciar que la presencia es relativamente reciente pues el $56,5 \%$ no supera los seis años de instauración; esta cifra es alentadora para la RSE ya que, en la constitución, como personas jurídicas, las empresas incorporan normas (estándares, certificaciones, regulaciones) que favorecen el desarrollo sustentable y protección del medioambiente, los cuales no eran consideradas como relevantes en épocas anteriores.

Tabla 2

Tiempo de presencia de las pyme en Salinas

\begin{tabular}{|l|c|c|}
\hline \multicolumn{1}{|c|}{ Tiempo } & Frecuencia & Porcentaje \\
\hline Menos de cuatro años & 4 & 17,4 \\
\hline De cuatro a seis años & 9 & 39,1 \\
\hline De siete a nueve años & 5 & 21,7 \\
\hline Más de nueve años & 5 & 21,7 \\
\hline Total & 23 & 100,0 \\
\hline
\end{tabular}

Fuente y elaboración: autores.

En cuanto al sector al que pertenecen las 23 empresas encuestadas, 12 pertenecen al sector servicios, 9 al sector industrial y 2 al comercio. El predominio de los servicios en el cantón Salinas se da principalmente en los 
servicios turísticos (hoteles y restaurantes) sustentado en las bondades que ofrece el cantón para el turismo nacional y extranjero.

La tabla 3 presenta los indicadores de gestión de la RSE de las pyme de Salinas, que corresponden a las 30 preguntas de la sección 5 de la encuesta. Para facilidad de análisis, las preguntas están ordenadas en forma descendente en función de las respuestas positivas dadas.

Tabla 3

\section{Existencia o práctica de indicadores de RSE por parte de las pyme de Salinas}

\begin{tabular}{|l|l|c|c|c|c|c|c|c|}
\hline No. & \multicolumn{1}{|c|}{ Tiempo } & Sí & $\%$ & No & $\%$ & $\begin{array}{c}\text { No } \\
\text { contesta }\end{array}$ & $\%$ & Total \\
\hline 16 & $\begin{array}{l}\text { Práctica del compromiso de la empresa } \\
\text { con el medioambiente }\end{array}$ & 23 & 100,0 & 0 & 0,0 & 0 & 0,0 & 23 \\
\hline 13 & $\begin{array}{l}\text { Presencia de protección, higiene } \\
\text { y prevención de riesgos laborales }\end{array}$ & 21 & 91,3 & 2 & 8,7 & 0 & 0,0 & 23 \\
\hline 19 & $\begin{array}{l}\text { Existencia de condiciones humanas } \\
\text { y ambiente saludable }\end{array}$ & 21 & 91,3 & 2 & 8,7 & 0 & 0,0 & 23 \\
\hline 14 & $\begin{array}{l}\text { Práctica de contribución a la salud } \\
\text { y a la afiliación al seguro social }\end{array}$ & 20 & 87,0 & 3 & 13.0 & 0 & 0,0 & 23 \\
\hline 29 & $\begin{array}{l}\text { Práctica de cumplimiento de leyes, } \\
\text { reglamentos y normas }\end{array}$ & 20 & 87,0 & 3 & 13,0 & 0 & 0,0 & 23 \\
\hline 2 & Práctica de principios éticos compartidos & 19 & 82,6 & 3 & 13,0 & 1 & 4,3 & 23 \\
\hline 17 & $\begin{array}{l}\text { Existencia de promoción } \\
\text { de la conciencia ambiental }\end{array}$ & 19 & 82,6 & 4 & 17,4 & 0 & 0,0 & 23 \\
\hline 4 & Práctica de inclusión social & 18 & 78,3 & 5 & 21,7 & 0 & 0,0 & 23 \\
\hline 6 & Existencia de prácticas anticorrupción & 18 & 78,3 & 5 & 21,7 & 0 & 0,0 & 23 \\
\hline 10 & $\begin{array}{l}\text { Existencia de eficiencia energética } \\
\text { y uso responsable del agua }\end{array}$ & 18 & 78,3 & 5 & 21,7 & 0 & 0,0 & 23 \\
\hline 15 & Práctica de capacitación continua & 18 & 78,3 & 5 & 21,7 & 0 & 0,0 & 23 \\
\hline 1 & Existencia de un código ético & 17 & 73,9 & 6 & 26,1 & 0 & 0,0 & 23 \\
\hline 8 & $\begin{array}{l}\text { Existencia de productos útiles } \\
\text { y condiciones justas a la sociedad }\end{array}$ & 17 & 73,9 & 4 & 17,4 & 2 & 8,7 & 23 \\
\hline 21 & $\begin{array}{l}\text { Práctica de atención y selección } \\
\text { de proveedores con responsabilidad } \\
\text { social }\end{array}$ & 17 & 73,9 & 5 & 21,7 & 1 & 4,3 & 23 \\
\hline 27 & $\begin{array}{l}\text { Existencia de transparencia } \\
\text { de las contribuciones }\end{array}$ & 17 & 73,9 & 5 & 21,7 & 1 & 4,3 & 23 \\
\hline
\end{tabular}


Percepción y práctica de la responsabilidad social empresarial: el caso de las pyme de Salinas

\begin{tabular}{|c|c|c|c|c|c|c|c|c|}
\hline 7 & $\begin{array}{l}\text { Práctica de distribución de la riqueza } \\
\text { generada }\end{array}$ & 16 & 69,6 & 6 & 26,1 & 1 & 4,3 & 23 \\
\hline 9 & Existencia de innovación tecnológica & 16 & 69,6 & 7 & 30,4 & 0 & 0,0 & 23 \\
\hline 25 & $\begin{array}{l}\text { Práctica de supervisión de la cadena } \\
\text { de suministro }\end{array}$ & 16 & 69,6 & 5 & 21,7 & 2 & 8,7 & 23 \\
\hline 18 & $\begin{array}{l}\text { Práctica de gestión del ciclo de vida } \\
\text { de productos y servicios }\end{array}$ & 15 & 65,2 & 7 & 30,4 & 1 & 4,3 & 23 \\
\hline 20 & $\begin{array}{l}\text { Práctica de lucha contra el cambio } \\
\text { climático }\end{array}$ & 15 & 65,2 & 8 & 34,8 & 0 & 0,0 & 23 \\
\hline 23 & $\begin{array}{l}\text { Existencia de competencia leal } \\
\text { y asociacionismo }\end{array}$ & 15 & 65,2 & 6 & 26,1 & 2 & 8,7 & 23 \\
\hline 5 & Práctica de rendición de cuentas & 14 & 60,9 & 9 & 39,1 & 0 & 0,0 & 23 \\
\hline 11 & $\begin{array}{l}\text { Presencia de gestión participativa } \\
\text { en la generación de productos y servicios }\end{array}$ & 14 & 60,9 & 8 & 34,8 & 1 & 4,3 & 23 \\
\hline 24 & Existencia de defensa de los ciudadanos & 14 & 60,9 & 7 & 30,4 & 2 & 8,7 & 23 \\
\hline 30 & $\begin{array}{l}\text { Práctica de atención al balance social } \\
\text { y a grupos en estado de vulnerabilidad }\end{array}$ & 14 & 60,9 & 8 & 34,8 & 1 & 4,3 & 23 \\
\hline 3 & $\begin{array}{l}\text { Existencia de gobernabilidad } \\
\text { y vinculación con accionistas }\end{array}$ & 12 & 52,2 & 10 & 43,5 & 1 & 4,3 & 23 \\
\hline 22 & $\begin{array}{l}\text { Existencia de actividades } \\
\text { con consumidores y clientes }\end{array}$ & 12 & 52,2 & 10 & 43,5 & 1 & 4,3 & 23 \\
\hline 28 & $\begin{array}{l}\text { Existencia de participación } \\
\text { en proyectos sociales }\end{array}$ & 12 & 52,2 & 10 & 43,5 & 1 & 4,3 & 23 \\
\hline 26 & $\begin{array}{l}\text { Existencia de participación } \\
\text { de los consumidores, comunidades } \\
\text { locales y grupos sociales }\end{array}$ & 9 & 39,1 & 13 & 56,5 & 1 & 4,3 & 23 \\
\hline 12 & $\begin{array}{l}\text { Existencia de relación con sindicatos } \\
\text { y agrupaciones gremiales }\end{array}$ & 2 & 8,7 & 21 & 91,3 & 0 & 0,0 & 23 \\
\hline
\end{tabular}

Fuente y elaboración: autores.

28 indicadores presentan respuestas positivas de más del 50\% de las empresas, lo que en general es un buen indicio en cuanto a la aplicación de la RSE en Salinas. De estos, cabe resaltar el indicador 16, "práctica del compromiso con el medioambiente", al cual la totalidad de las empresas encuestadas respondió que sí tienen buenas prácticas de conservación ambiental. Igualmente, son altas las prácticas de protección, higiene y prevención de riesgos laborales y de condiciones humanas y ambiente saludable con un $91 \%$ de respuestas positivas. Estos resultados permiten ver que hay cierta predisposi- 
ción positiva de las empresas, principalmente con el medioambiente, seguido por la atención a los actores internos, aunque en este último grupo -indicador 12 "existencia de relación con sindicatos y gremios"- el 91\% de las pyme respondió que no existe una relación con sindicatos, lo cual significa que casi todas las pyme de Salinas no favorecen la asociatividad laboral al interior de las empresas, otro indicador que fue calificado positivamente por el $39 \%$ de las pyme fue el 26 sobre la "existencia de participación de los consumidores, comunidades locales y grupos sociales", lo cual implica cierta desconexión de las empresas con el público externo en cuanto al relacionamiento con estos actores en actividades participativas y comunitarias.

En cuanto a la RSE y su vínculo con el desarrollo humano, la tabla 4 muestra en qué medida las pyme de Salinas participan en tareas de RSE y si contribuyen o no al desarrollo humano.

Tabla 4

Participación en RSE y contribución al desarrollo humano

\begin{tabular}{|l|c|c|c|c|c|c|c|c|c|c|c|c|}
\cline { 2 - 12 } \multicolumn{1}{c|}{} & \multicolumn{2}{c|}{ Mucho } & \multicolumn{2}{c|}{ Bastante } & \multicolumn{2}{c|}{ Poco } & \multicolumn{2}{c|}{ Nada } & \multicolumn{2}{c|}{ No sabe } & \multicolumn{2}{c|}{ Total } \\
\cline { 2 - 11 } \multicolumn{1}{c|}{} & No. & $\%$ & No. & $\%$ & No. & $\%$ & No. & $\%$ & No. & $\%$ & No. & $\%$ \\
\hline $\begin{array}{l}\text { Participación } \\
\text { en tareas } \\
\text { de RSE }\end{array}$ & 2 & 8,7 & 5 & 21,7 & 13 & 56,5 & 1 & 4,4 & 2 & 8,7 & 23 & 100 \\
\hline $\begin{array}{l}\text { Contribución } \\
\text { al desarrollo } \\
\text { humano }\end{array}$ & 10 & 43,4 & 6 & 26,1 & 6 & 26,1 & 0 & 0 & 1 & 4,4 & 23 & 100 \\
\hline
\end{tabular}

Fuente y elaboración: autores.

De estos resultados se desprende que un $70 \%$ de las empresas tienen una importante contribución al desarrollo humano, mientras que solo un 30\% participan en tareas de RSE, lo cual permite inferir que las pyme de Salinas no necesariamente vinculan las prácticas de RSE con el desarrollo humano y más bien existiría la percepción que su quehacer empresarial de por sí aporta al desarrollo humano sea por medio de la provisión de oportunidades laborales, el cumplimiento de las leyes laborales, el trato a sus trabajadores, entre 
otros aspectos que para el empresario promedio son muchas veces necesarios y suficientes para aportar al desarrollo humano.

Esto en alguna medida es coherente con el hecho de que existen empresas cuya cultura empresarial, valores organizacionales y liderazgo de mercado son condiciones que les permiten responder en forma socialmente responsable y, por ende, contribuir a mejorar las condiciones de vida de sus empleados y colaboradores, así como propender al desarrollo comunitario y de la sociedad como un todo: "En tal sentido, son empresas en las que el concepto y alcance de la responsabilidad social no están plenamente arraigados" (Torresano 2012, 19).

\section{Percepción de la población}

En la tabla 5 se presentan las respuestas de los pobladores por parroquia a la pregunta de si alguna vez han escuchado hablar de la RSE.

Tabla 5

Conocimiento sobre la RSE

\begin{tabular}{|c|c|c|c|c|c|c|c|c|}
\hline \multirow{2}{*}{ Respuestas } & \multicolumn{6}{|c|}{ Estrato } & \multirow{2}{*}{ Total } & \multirow{2}{*}{$\%$} \\
\hline & Salinas & $\mathrm{Fr}^{*}$ & $\begin{array}{c}\text { J. L. } \\
\text { Tamayo }\end{array}$ & $\mathrm{Fr}^{*}$ & Anconcito & $\mathrm{Fr}^{*}$ & & \\
\hline Sí & 48 & 0,63 & 15 & 0,20 & 13 & 0,17 & 76 & 49 \\
\hline No & 29 & 0,37 & 37 & 0,47 & 13 & 0,16 & 79 & 51 \\
\hline Total & 77 & 0,50 & 52 & 0,33 & 26 & 0,17 & 155 & 100 \\
\hline
\end{tabular}

*Frecuencia.

Fuente y elaboración: autores.

Los resultados muestran que el 49\% respondió que sí conoce o está familiarizado con la RSE frente al 51\% que manifestó desconocer por completo del tema. Por parroquia, Salinas demuestra estar más familiarizado con el tema ya que el $63 \%$ respondió afirmativamente, comparado con José Luis 
Tamayo 20\% y Anconcito 17\% respectivamente, que afirmaron haber escuchado el término. Se puede colegir, entonces, que las condiciones de vida, base para la estratificación, propician diferencias significativas respecto del dominio, comprensión o conocimiento sobre la RSE.

Es de esperar que la percepción que se tenga de la RSE esté ligada a la consideración que el individuo tenga como actor social. Por ejemplo, si una persona en su valoración estima que es un consumidor/cliente en el tejido social, entonces, apreciará que la empresa ponga a su disposición un bien o servicio de calidad; además, que en la producción del mismo agregue transparencia, ética, respeto a la norma y el medioambiente. Otro aspecto que observa en el momento de la compra es que, en la presentación del bien o servicio, estén identificadas claramente las garantías y bondades que el mismo ofrece.

La tabla 6 muestra los resultados sobre qué tipo de actor social se consideran los encuestados. El 26,5\% declaró ser consumidor o cliente. De este $26,5 \%$, el $44 \%$ pertenece a Salinas, el 32\% a José Luis Tamayo y el 24\% a Anconcito. Parece ser que, el actor social que se define como consumidor/ cliente sabe lo que espera del bien o servicio; es decir, que, en primer lugar, satisfaga sus necesidades y también, que el producto garantice su uso o para lo que se lo compra; es decir, que una empresa se responsabilice de lo que hace y como lo hace.

Tabla 6

Tipo de actor social

\begin{tabular}{|l|c|c|c|c|c|c|c|c|}
\hline \multirow{2}{*}{ Actor social } & \multicolumn{6}{|c|}{ Estrato } & \multirow{2}{*}{ Total } & \multirow{2}{*}{$\%$} \\
\cline { 2 - 10 } & Salinas & Fr* $^{*}$ & $\begin{array}{c}\text { J. L. } \\
\text { Tamayo }\end{array}$ & fr & Anconcito & $\mathbf{F r}^{*}$ & & \\
\hline Comunidad & 31 & 0,48 & 22 & 0,34 & 12 & 0,18 & 65 & 41,9 \\
\hline Consumidor/cliente & 18 & 0,44 & 13 & 0,32 & 10 & 0,24 & 41 & 26,5 \\
\hline Empleado & 17 & 0,52 & 12 & 0,36 & 4 & 0,12 & 33 & 21,3 \\
\hline Empresario & 11 & 0,69 & 5 & 0,31 & 0 & 0,00 & 16 & 10,3 \\
\hline Total & 77 & 0,50 & 52 & 0,33 & 26 & 0,17 & 155 & 100,0 \\
\hline
\end{tabular}

*Frecuencia.

Fuente y elaboración: autores. 
En cuanto a la población vista como comunidad, es decir, cuando deja de ser individuo para formar parte de un colectivo, el 41,9\% declaró ser parte de la comunidad; de este, el 48\% reside en Salinas, el 34\% en José Luis Tamayo y el $18 \%$ en Anconcito.

Cuando las personas se declaran parte de un colectivo, sus necesidades, deseos y aspiraciones individuales se convierten en parte de un todo. En este sentido, la RSE debe actuar de manera distinta; es decir, la actividad productiva tiene que ser complementada con acciones que beneficien al conglomerado; ya no se trata de producir un bien o servicio con las mejores prestaciones posibles, sino también de contribuir con la comunidad en salud, educación y protección del medioambiente.

El estigma de que empleador y empleado o colaborador son fuerzas contrarias en el proceso de producción y, por lo tanto, en constante desavenencia, es cosa del pasado para toda visión de la RSE; hoy, estas fuerzas son complementarias, es decir, las estrategias vigentes son remar en una misma dirección, utilizando solo una carta de navegación.

La cualidad de empleado o colaborador arrojó que el 21,3\% de los encuestados corresponden a esta modalidad; de este, el 52\% vive en Salinas; lo que refleja que el mayor número de empresas tiene su asentamiento en la ciudad de Salinas; el 36\% reside en José Luis Tamayo y el 12\% en Anconcito. Estas cifras son favorables para la RSE pues la gestión social responsable puede extenderse a todos los habitantes del cantón sin intermediarios o inconvenientes de logística.

Sin importar cuál sea o haya sido el modelo, modo de producción o generación de riqueza, el esfuerzo del individuo emprendedor e innovador ha sido una constante. Sin embargo, convertirse en empresario no es una tarea fácil; se requiere de un espíritu y cualidades diferenciadas. De ahí que solo el 10,3\% de los involucrados en la encuesta se identificó como empresario; de este porcentaje, el 69\% pertenece a Salinas, 31\% a José Luis Tamayo, mientras que Anconcito no registra a actores sociales que se identifiquen como empresarios.

Los resultados permiten vislumbrar que, en la medida en que la población se perciba como un ente colectivo, existe un entorno favorable para que la gestión empresarial promueva proyectos y planes de acción generadores de riqueza y bienestar para la comunidad. 
La gestión vinculada del Estado, la empresa y la sociedad civil genera un agregado y potencia los resultados de la gestión de la RSE; obviamente la credibilidad y transparencia de los procedimientos de las organizaciones públicas y privadas deben ser parte de la estrategia de responsabilidad empresarial. Por su parte, el Estado tiene que generar confianza en la empresa, brindar seguridad jurídica, reglas de juego diáfanas y de largo alcance. Se trata entonces de que el Estado, la organización privada y la sociedad civil juntos generen valor social.

En la búsqueda de una respuesta por parte de la sociedad civil sobre quién debería asumir un rol preponderante o principal en la consecución de propósitos específicos que coadyuven a la responsabilidad social, los encuestados respondieron según se muestra en la tabla 7 a continuación.

Tabla 7

Asignación de responsabilidades en los roles de los sectores público y privado

\begin{tabular}{|c|c|c|c|c|c|c|c|}
\hline \multirow{2}{*}{ Roles } & \multicolumn{6}{|c|}{ Sectores } & \multirow{2}{*}{ Total } \\
\hline & Estado & $\%$ & Empresa & $\%$ & Ambos & $\%$ & \\
\hline $\begin{array}{l}\text { Promover la educación } \\
\text { y el crecimiento personal }\end{array}$ & 60 & 38,7 & 6 & 3,9 & 89 & 57,4 & 155 \\
\hline $\begin{array}{l}\text { Compromiso con } \\
\text { el medioambiente }\end{array}$ & 29 & 18,7 & 28 & 18,1 & 98 & 63,2 & 155 \\
\hline Promover proyectos comunitarios & 52 & 33,5 & 10 & 6,5 & 93 & 60,0 & 155 \\
\hline Promover la inclusión social & 62 & 40,0 & 14 & 9,0 & 79 & 51,0 & 155 \\
\hline $\begin{array}{l}\text { Contribuir a disminuir la brecha } \\
\text { entre ricos y pobres }\end{array}$ & 63 & 40,7 & 12 & 7,7 & 80 & 51,6 & 155 \\
\hline $\begin{array}{l}\text { Informar al público de las prácticas } \\
\text { sociales y medioambientales }\end{array}$ & 48 & 31,0 & 16 & 10,3 & 91 & 58,7 & 155 \\
\hline
\end{tabular}

Fuente y elaboración: autores.

Con relación a promover la educación y el crecimiento personal: el pronunciamiento de la población a esta interrogante fue: 38,7\% responsabiliza al Estado como el actor principal en la gestión de educar a la población; 3,9\% responsabiliza a la empresa privada; y el 57,4\% a ambos. 
Compromiso con el medioambiente: a más de la educación, la implementación de compromisos como la protección del medioambiente es otro componente a tener en cuenta a la hora de asumir la gestión de responsabilidad social. Para la población objeto de estudio la principal carga recae en ambos con un $63,2 \%$. Nuevamente, la población se pronuncia por una labor en conjunto; es decir, no les convence los esfuerzos aislados.

Promover proyectos comunitarios: la identidad de la comunidad se manifiesta cuando se funda en el colectivo y, a través de este, emite una opinión o construye una demanda social plasmada en proyectos comunitarios. El resultado de este cuestionamiento es responsabilizar principalmente a ambos actores en forma mancomunada con el $60 \%$, seguido por el Estado con el $33,5 \%$ y solo la empresa con un $6,5 \%$.

Promover la inclusión social: una característica de los seres vivientes es estar en constante movimiento; por lo tanto, aspirar a ser parte activa de la dinámica social, cultural, política y económica de todo actor social, ha sido una perseverancia en el devenir de los pueblos. En este sentido, la investigación sobre quién o quienes deberían asumir un rol preponderante en la promoción de la inclusión social, la sociedad civil respondió de la siguiente manera: Estado, 40\%; empresa, 9\% y ambos, $51 \%$. Al igual que en los cuestionamientos anteriores, la población considera que es una responsabilidad que debe ser asumida conjuntamente por el Estado y por la empresa.

Contribuir a disminuir la brecha entre ricos y pobres: en el proceso de acumulación de excedentes, el esfuerzo del individuo siempre ha sido la primera opción; es decir, es este quien decide cuánto hacer o tener. Sin embargo, cuando las oportunidades son escasas y los recursos limitados, es necesario contar con alternativas u opciones orientadas por terceros. En esta premisa, la población espera que la orientación provenga principalmente de la acción conjunta de ambos actores $51,6 \%$, seguida por el Estado, 40,6\% y finalmente la empresa, $7,7 \%$.

Informar el público de las prácticas sociales y medioambientales: la Carta Magna en vigencia, demanda una rendición de cuentas de todos los actores sociales; es decir, a más de asumir la responsabilidad de los actos, también se debe informar cómo se están haciendo las cosas; en este sentido, el pronunciamiento de la sociedad civil es nuevamente asignar mayor responsabilidad a los dos actores en conjunto 58,7\%, luego al Estado con un $31 \%$ y la empresa con el 10,3\%. 
El criterio de la sociedad civil entonces es que las acciones en pro del bienestar de los pueblos deben ser una tarea conjunta gobierno-empresa privada y no un esfuerzo aislado. Partiendo de este aspiración, surge la siguiente cuestión: ¿Quién hace qué? Es decir, se necesita a un Estado gestor y a una empresa hacedora, a un Estado que sea juez y parte o a un Estado que intervenga donde el esfuerzo privado no puede llegar.

Si bien la sociedad civil concibe la responsabilidad compartida como eje fundamental de la RSE, no es menos cierto que la empresa es la entidad gestora por excelencia del cambio social y, por ende, el ciudadano común siente que las prácticas empresariales influyen directamente en su bienestar individual y colectivo así como en el medioambiente. Por esto es importante conocer cómo la sociedad civil valora las prácticas empresariales con relación a los principales indicadores de RSE. Al respecto, la tabla 8 muestra dicha valoración.

Tabla 8

Valoración de la práctica empresarial

\begin{tabular}{|l|c|c|c|c|c|c|c|c|c|}
\hline \multicolumn{1}{|c|}{ Aspectos a valorar } & Excelente & $\%$ & Buena & $\%$ & Regular & $\%$ & Mala & $\%$ & Total \\
\hline $\begin{array}{l}\text { Calidad de la producción } \\
\text { y los servicios }\end{array}$ & 18 & 11,6 & 74 & 47,7 & 55 & 35,5 & 8 & 5,2 & 155 \\
\hline Cuidado al medioambiente & 7 & 4,5 & 61 & 39,4 & 74 & 47,7 & 13 & 8,4 & 155 \\
\hline $\begin{array}{l}\text { Desarrollo de proyectos } \\
\text { sociales y comunitarios }\end{array}$ & 10 & 6,5 & 59 & 38,1 & 71 & 45,8 & 15 & 9,7 & 155 \\
\hline $\begin{array}{l}\text { Atención al consumidorl } \\
\text { cliente }\end{array}$ & 20 & 12,9 & 79 & 51,0 & 49 & 31,6 & 7 & 4,5 & 155 \\
\hline $\begin{array}{l}\text { Atención integral } \\
\text { al trabajador }\end{array}$ & 8 & 5,2 & 78 & 50,3 & 55 & 35,5 & 14 & 9,0 & 155 \\
\hline $\begin{array}{l}\text { Aporte de productos útiles } \\
\text { en condiciones justas }\end{array}$ & 18 & 11,6 & 72 & 46,5 & 51 & 32,9 & 14 & 9,0 & 155 \\
\hline $\begin{array}{l}\text { Valoración promedio } \\
\text { porcentual }\end{array}$ & & 8,7 & & 45,5 & & 38,2 & & 7,6 & $100 \%$ \\
\hline
\end{tabular}

Fuente y elaboración: autores. 
De acuerdo con los resultados, en promedio, un $84 \%$ de los encuestados valoraron la práctica empresarial entre buena y regular. Es de entender que la gestión de RSE en el cantón Salinas no ha adquirido un reconocimiento destacado por parte de la sociedad civil hasta el momento; es decir, no se vislumbra una gestión explícita de la empresa en beneficio del actor social, de tal manera que este valore su real presencia para con el entorno, la comunidad y el medioambiente.

Finalmente, la opinión de los ciudadanos con relación al aporte de las empresas al desarrollo humano, se obtuvo como resultado que el 51\% considera que las empresas aportan poco al desarrollo humano de esta parte del país y el $7 \%$ considera que las empresas no aportan nada; $22 \%$ contestaron que aportan entre mucho y bastante y el 20\% restante no sabían. Esto sugiere que la población en general tiene una imagen no muy favorable sobre la contribución de la empresa al desarrollo humano, lo cual corrobora de alguna forma el punto de vista de Friedman (1970) en que el rol de la empresa es netamente económico y cualquier acción altruista se sustenta en el individuo accionista o propietario, criterio al parecer compartido por la gente.

\section{Conclusiones}

Las pyme del cantón Salinas, en su mayoría jóvenes, reflejan en sus prácticas empresariales consciencia respecto al hecho de actuar de manera social y ambientalmente responsable. Sin embargo, se concluye que la gestión empresarial no siempre responde a una comprensión cabal y práctica de la RSE, sino que en su quehacer diario del relacionamiento con sus colaboradores, sus clientes, sus proveedores y la comunidad en general, el empresario siente que actúa de manera coherente a lo que se espera de su rol como actor social, esto es, responsables con sus compromisos laborales, con el medioambiente, cumplidores de las leyes y de los principios éticos.

En tal sentido, es importante crear mayor consciencia y conocimiento sobre la RSE en el entorno empresarial de Salinas como una forma de que la gestión de las empresas tenga mayor efectividad y trascienda con resultados visiblemente positivos hacia la comunidad en un ambiente de justicia y equidad, propicio para crear las bases de un verdadero desarrollo humano. 
Por el lado de la población, la responsabilidad social es un concepto familiar en la mayoría de los pobladores de la cabecera cantonal de Salinas no así en las otras parroquias del estudio donde sigue siendo un concepto elusivo; en parte, esto se explica por las diferentes condiciones de vida entre los estratos del estudio.

La ciudadanía del cantón Salinas en general percibe que el accionar de la empresa como gestora de cambio social y por ende que contribuye positivamente al desarrollo humano es aún incipiente. Cualquier esfuerzo privado es en cierto modo visto como una acción que recae netamente en la decisión individual del propietario más que en principios de la organización.

Es de esperar entonces, que sean los esfuerzos conscientes y con conocimiento de causa los que lleven a las empresas a actuar de manera coherente con los principios de la RSE, de manera que los ciudadanos valoren la presencia y práctica empresarial en base a acciones y resultados tangibles en términos del desarrollo de la comunidad y el entorno.

Salinas y el resto de la provincia constituyen un campo propicio para promover la RSE y propender a mejores bases para su desarrollo humano.

\section{Referencias}

Barco Sousa, José Manuel. 2015. "RSE y creación de valor compartido". Consulta: abril de 2016. 〈http://www.responsabilidadsocialempresarial.com/? $\mathrm{p}=346$ 〉.

Cancino, Christian, y Mario Morales. 2008. "Responsabilidad social empresarial". Serie Documento Docente, No. 1: 1-58. Consulta: marzo de 2016. 〈http://repositorio.uchile.cl/bits tream/handle/2250/122747/Cancino_Morales_2008.pdf?sequence $=1$.

Canessa Illich, Giuliana, y Emilio García Vega. 2005. El ABC de la responsabilidad social empresarial en el Perú y en el mundo. Lima: Perú 2021. Consulta: abril de 2016. «http:// www.peru2021.org/repositorioaps/0/0/par/elabcperu2021/abc\%20de\%201a\%20rse\%20 en\%20el\%20per\%C3\%BA\%20y\%20el\%20mundo.pdf .

Cochran, William G. 1991. Técnicas de muestreo. Ciudad de México: Compañía Continental. Fraga, Rafael, Caridad Herrera y Diego Gómez. 2015. "Indicadores de responsabilidad social para pymes del cantón Salinas y su incidencia en el desarrollo humano". Revista Economía y Negocios 6, No. 1: 28-41.

Friedman, Milton. 1970. "The Social Responsibility of Business is to Increase its Profits". Documento de trabajo. Consulta: marzo de 2016. 〈http://link.springer.com/chapter/ 10.1007/978-3-540-70818-6_14>. 
Percepción y práctica de la responsabilidad social empresarial: el caso de las pyme de Salinas

Garriga, Elisabet, y Domènec Melé. 2004. "Corporate Social Responsibility Theories: Mapping the Territory". Journal of Business Ethics 53: 52-71.

Instituto Nacional de Estadística y Censos (INEC). 2010. VII Censo de Población y VI de Vivienda. Quito: INEC. Consulta: marzo de 2016. «http://anda.inec.gob.ec/anda/index.php/ catalog/270).

International Organization for Standardization (ISO). 2010. ISO 26000: guía de responsabilidad social. Consulta: abril de 2016. 〈https://www.iso.org/iso-26000-social-responsibility. html.

Moon, Jeremy, y David Vogel. 2008. "Corporate Social Responsibility, Government, and Civil Society". En Andrew Crane y otros, edit., The Oxford Handbook of Corporate Social Responsibility. 3-20. Nueva York: Oxford University Press.

Programa de las Naciones Unidas para el Desarrollo (PNUD). 2015. Human Development Reports. ¿Qué es el Desarrollo Humano? Washington DC: Communications Development Incorporated. Consulta: mayo de 2017. 〈http://hdr.undp.org/es/content $/ \% \mathrm{C} 2 \% \mathrm{BFqu} \% \mathrm{C}$ 3\%A9-es-el-desarrollo-humano».

Secretaría Nacional de Planificación y Desarrollo (SENPLADES). 2013. Plan Nacional para el Buen Vivir 2013-2017. Quito: SENPLADES. Consulta: septiembre de 2016. «http:// www.buenvivir.gob.ec/s.

Torresano, Mónica. 2012. Estudio de responsabilidad social de empresas del Ecuador. Fundación Avina / Cooperación Alemana al Desarrollo GIZ / IDE Business School. Consulta: septiembre de 2016. hhttp://responsabilidadsocialquito.com.ec/wp-content/ uploads/2015/09/Estudio-de-RS-de-empresas-del-Ecuador-2012.pdf〉. 